\title{
COMMENSURABILITY AND THE CHARACTER VARIETY
}

\author{
D.D. LONG AND A.W. REID
}

\section{Introduction}

Recall that hyperbolic 3-manifolds $M$ and $N$ are said to be commensurable if they have a common finite sheeted covering. This is equivalent to the fundamental groups having subgroups of finite index which are conjugate in $\operatorname{PSL}(2, \mathbf{C})$. In general it is very difficult to determine if two manifolds are commensurable or not, once the most obvious invariants of commensurability (for example, the invariant trace field, see [13] and [17]) agree. When $M$ is a finite volume hyperbolic 3-manifold with a single cusp, its $\operatorname{SL}(2, \mathbf{C})$-representation and character varieties, denoted respectively, by $R(M)$ and $X(M)$ throughout, have been fundamental tools in understanding the topology of $M$, see [6], [5], and [4]. These techniques can be extended to the $\operatorname{PSL}(2, \mathbf{C})$-character variety of $M$, which we denote by $Y(M)$ ([2], and see $\S 2.1$ for some details). Throughout, for either $\mathrm{SL}(2)$ or PSL(2), we use the subscript 0 to denote a component of $X(M)$ (or $Y(M)$ ) containing the character of a faithful discrete representation of $\pi_{1}(M)$. The main results of this paper concern how $Y_{0}(M)$ be can used to detect incommensurability. For example, one of the main results can be summarized in the following (for terminology and definitions see $\S 2$ ):

Theorem 1.1. Suppose that $M_{1}$ and $M_{2}$ are 1-cusped hyperbolic 3-manifolds that cover a common orbifold with a flexible cusp.

Then $Y_{0}\left(M_{1}\right)$ is birational to $Y_{0}\left(M_{2}\right)$.

In the case when the manifolds are non-arithmetic, the work of Margulis (see [11]) shows that the commensurability class contains a unique minimal element and we get the more succinct re-formulation about the entire commensurability class of $M$ :

Theorem 1.2. Suppose that $M$ is a non-arithmetic 1-cusped hyperbolic 3-manifold for which the minimal element in the commensurability class has a flexible cusp.

Then $Y_{0}(M)$ is an invariant of the commensurability class of $M$.

Received June 21, 1999.

This work was partially supported by the N. S. F., The Alfred P. Sloan Foundation and T. A. R. P. The 2nd author also wishes to thank the Department of Mathematics at U.C.S.B. for its hospitality whilst working on the contents of this paper. 
One can often verify this condition directly, for example, a rigid cusp places constraints on the invariant trace field:

Theorem 1.3. Suppose that $M$ is a non-arithmetic, 1-cusped hyperbolic 3-manifold whose invariant trace field does not contain either $\mathbf{Q}(\sqrt{-1})$ or $\mathbf{Q}(\sqrt{-3})$.

Then $Y_{0}(M)$ is an invariant of the commensurability class of $M$.

Since the genus of a smooth projective curve is an invariant of birational equivalence ([12] Chapter 7) we deduce,

Corollary 1.4. Suppose that $M$ is a non-arithmetic 1-cusped hyperbolic 3-manifold as in 1.2 or 1.3.

Then the genus of the smooth model of $Y_{0}\left(M_{1}\right)$ is an invariant of the commensurability class of $M$.

The proof of Theorem 1.1 is contained in $\S 3$, as are further results linking commensurability with boundary slopes.

We remark that the theorem is false for the $\mathrm{SL}(2, \mathbf{C})$-character variety, as we illustrate in $\S 4$. The final section contains a discussion of examples, in particular we exhibit a pair of manifolds with the same invariant trace fields and volume which have $\operatorname{PSL}(2, \mathbf{C})$-character varieties of different genus and so cannot be commensurable.

All hyperbolic manifolds and orbifolds are assumed orientable and finite volume.

\section{Acknowledgements}

We would like to thank Fernando Rodriguez-Villegas for some helpful conversations, and in particular for supplying Theorem 4.1. We also wish to thank the referee for their careful reading of the original version of this paper and for many useful comments.

\section{Preliminaries}

2.1. The $\operatorname{PSL}(2, \mathbf{C})$-character variety. For background on the $\operatorname{SL}(2, \mathbf{C})$ character variety we refer the reader to [6] or [5] Chapter 1 . There is also a notion of a $\operatorname{PSL}(2, \mathbf{C})$-character variety for a finitely presented group $G$. This is a good deal less well-known, and its construction not quite as standard. We briefly recall a construction, see also [2] and [9] for closely related versions.

Let $G$ be a finitely generated group. The $\mathbf{Z}_{2}$-central extensions of $G$ are classified by the finite group $H^{2}\left(G ; \mathbf{Z}_{2}\right)$. For each such cohomology class $u$, we form a central extension $G_{u}$ which is unique up to isomorphism. Let $X\left(G_{u}\right)$ be the $\operatorname{SL}(2, \mathbf{C})$-character variety of the group $G_{u}$; this is an affine algebraic set which admits a natural action of the group $H^{1}\left(G_{u} ; \mathbf{Z}_{2}\right)$, namely

$$
\epsilon\left(\chi_{\rho}\right)(\gamma)=\chi_{\epsilon(\rho)}(\gamma)=\epsilon(\gamma) \chi_{\rho}(\gamma)
$$

where $\epsilon \in H^{1}\left(G_{u} ; \mathbf{Z}_{2}\right), \chi_{\rho} \in X\left(G_{u}\right)$ and $\gamma \in G_{u}$. 
This action is algebraic, and permutes irreducible components of $X\left(G_{u}\right)$ and the quotient space $X\left(G_{u}\right) / H^{1}\left(G_{u} ; \mathbf{Z}_{2}\right)$ can be given the structure of an affine algebraic set. The union of all these algebraic sets as $u$ runs over elements of $H^{2}\left(G ; \mathbf{Z}_{2}\right)$ is the $\operatorname{PSL}(2, \mathbf{C})$-character variety of the group $G$.

The construction of a $\operatorname{PSL}(2, \mathbf{C})$-representation variety is entirely analogous.

Example: The knot $5_{2}$. The knot $5_{2}$ is a twist knot with 2-bridge normal form $(7,5)$. A presentation for the fundamental group of the complement is:

$$
\left\langle a, b \mid w a w^{-1}=b, w=a^{-1} b a^{-1} b^{-1} a b^{-1}\right\rangle .
$$

Mathematica can be used to determine the defining polynomial equation for $X_{0}$ (see $[10])$ :

$$
s(z, t)=1+2 t-t^{2}-t^{3}+\left(-t+t^{2}\right) z^{2},
$$

where $z$ is the trace of the meridional element $a$ (which is the same as that of $b)$ and $t$ is the trace of $a b^{-1}$. There is a rather obvious birational change of cocordinates which puts the curve in the form $y^{2}=f(t)$ with $f(t)=\left(1+2 t-t^{2}-\right.$ $\left.t^{3}\right)\left(t-t^{2}\right)$, a polynomial with distinct roots. This curve (or rather the smooth model of the projective completion) is then hyperelliptic of genus 2 (see [10]).

For all knots in $S^{3}$, representations come in pairs. In this case, the action of $H^{1}\left(\pi_{1}(M) ; \mathbf{Z}_{2}\right) \cong \mathbf{Z}_{2}$ on the character variety is given by $(z, t) \rightarrow(-z, t)$ so that we can identify the component $Y_{0}$ of the $\operatorname{PSL}(2, \mathbf{C})$-character variety with the zero set of

$$
p(q, t)=1+2 t-t^{2}-t^{3}+\left(t^{2}-t\right) q .
$$

The affine curve $p(q, t)=0$ is a punctured sphere, since it is easy to see from the equation that $q$ is a rational function of $t$. Hence the smooth model of the projective completion of $Y_{0}$ is a sphere. Indeed the covering of smooth models $\widehat{X_{0}} \rightarrow \widehat{Y_{0}}$ is a hyperelliptic covering of a genus 2 surface branched over the sphere.

2.2. Dehn surgery on orbifolds : flexible and rigid cusps of orbifolds. For more details on the contents of this section see [19], [14] and [8].

If $Q$ is a finite volume hyperbolic 3-orbifold with a single cusp, then the cusp end of the orbifold has the form $T \times[0, \infty)$ where $T$ is an orientable Euclidean 2-orbifold. The possibilities for $T$ are $T^{2}$, a pillowcase or a turnover, see [8]. Recall by a pillowcase we mean a sphere with 4 cone points of cone angle $\pi$. We shall henceforth denote this orbifold by $P$. By a turnover we mean a sphere with 3 cone points with cone angles one of

$$
\{(\pi, \pi / 2, \pi / 2),(2 \pi / 3,2 \pi / 3,2 \pi / 3),(\pi, 2 \pi / 3, \pi / 3)\},
$$

corresponding to quotients of the Euclidean plane by the triangle groups $(2,4,4)$, $(3,3,3)$ and $(2,3,6)$ respectively.

Now triangle groups are well known to be rigid in that they admit a finite number of $\operatorname{PSL}(2, \mathbf{C})$-representations up to conjugacy. The pillowcase, $P$ is flexible, in the sense that it admits many distinct Euclidean structures. We call a cusp of a 3-orbifold rigid if a horospherical cross-section of the cusp end is a turnover, and flexible if it is a torus or a pillowcase. 
This dichotomy manifests itself in defining Dehn surgery on cusps of hyperbolic 3-orbifolds. We briefly recall the details, see [8] for more.

Let $Q$ be a cusped hyperbolic 3-orbifold. If a cusp of $Q$ is flexible, Dehn surgery may be performed as follows. In the case where a horospherical crosssection is a torus, one proceeds as in the case of a manifold. For the pillowcase we proceed as follows. There is a canonical involution $\tau: T^{2} \rightarrow T^{2}$ acting as -1 on $H_{1}\left(T^{2} ; \mathbf{Z}\right)$, and which defines an orbifold covering map $\pi: T^{2} \rightarrow P$. The involution $\tau$ extends to a map between the solid torus, and so $\pi$ extends to a map between the solid torus and the solid pillowcase. By choosing a homology basis for the 2 -fold cover of $P$ we can define $p / q$-surgery on the end $P \times[0, \infty)$ to mean cutting off the end and regluing it in a way that induces $p / q$-surgery on the 2 -fold cover of the end. This corresponds to attaching a disc to a $p / q$-curve $\gamma$ say, in the 2-fold cover of the end so that under the map $\pi, \gamma$ projects to a power of a simple loop in $P$. Following Thurston ([19], [14] and also [15] for some corrections), this extension holds for generalized hyperbolic Dehn surgeries on a pillowcase end of an orbifold.

There is no non-trivial Dehn surgery on a rigid cusp, the point of difference being that no solid torus quotient has a turnover as boundary — since any self-homeomorphism of a solid torus takes meridians to meridians.

As in the case of manifolds, Thurston's generalized hyperbolic Dehn surgery space is closely related to the components $X_{0}$ and $Y_{0}$ of the appropriate character varieties. Roughly, we associate to a point of the hyperbolic Dehn surgery space a holonomy representation into $\operatorname{PSL}(2, \mathbf{C})$, and then take its character. Thus, as in the case of manifolds the following can be deduced from the existence of hyperbolic deformations in the Dehn surgery space, see [8].

Theorem 2.1. Let $Q$ be a 1-cusped hyperbolic 3-orbifold of finite volume. If $Q$ has a rigid cusp then $Y_{0}(Q)$ is a single point, and if $Q$ has a flexible cusp then $\operatorname{dim}_{\mathbf{C}}\left(Y_{0}\right)=1$.

\section{Remarks.}

(i) The definition of $Y_{0}$ is unambiguous because the character of a discrete faithful representation is a smooth point and therefore lies on a unique component.

(ii) When $Q$ has more than one cusp an analogous statement holds. In this case, the dimension of $Y_{0}$ is the same as the number of flexible cusps of $Q$ (see $[8])$.

\section{Main results}

3.1. The key tool in our analysis is the following simple lemma. To make the cleanest statement we define a representation $\rho$ of a group $G$ into $\operatorname{SL}(2, \mathbf{C})$ to be strongly irreducible if the image group $\rho(G)$ contains free group of rank two. Note that strongly irreducible representations are always in particular irreducible. The same definition will be used in the context of $\operatorname{PSL}(2, \mathbf{C})$-representations. Irreducible representations which are not strongly irreducible are fairly rare (the lift 
of the irreducible representation of the infinite dihedral group from $\operatorname{PSL}(2, \mathbf{C})$ to $\mathrm{SL}(2, \mathbf{C})$ is an example) and it follows, for example, from the results of $[6]$ that if a component of the character variety contains the character of a strongly irreducible representation, then the characters of strongly irreducible representations are Zariski open.

Lemma 3.1. If two representations of a group $G$ into $\mathrm{SL}(2, \mathbf{C})$ are strongly irreducible on a subgroup of finite index and agree up to signs, then they agree up to signs on $G$.

Proof. Let the representations be $\rho_{1}$ and $\rho_{2}$ and the subgroup of finite index given by the hypothesis be denoted by $F$.

Note that by passing to the kernel of the map $F \rightarrow H_{1}\left(F ; \mathbf{Z}_{2}\right)$, we may as well assume that the representations agree exactly on a subgroup of finite index; we denote the common value of the representations by $\rho$. By passage to another subgroup of finite index if necessary, we may as well suppose that this latter subgroup, $K$ say, is normal in $G$. We note that the hypothesis of strong irreducibility guarantees that $\rho$ continues to be irreducible when restricted to the normal subgroup $K$.

Let $g \in G$ and consider any element $k \in K$. Then

$$
\rho_{1}(g) \rho_{2}\left(g^{-1}\right) \rho(k) \rho_{2}(g) \rho_{1}\left(g^{-1}\right)=\rho_{1}(g) \rho_{2}\left(g^{-1} k g\right) \rho_{1}(g),
$$

and since $g^{-1} \mathrm{~kg}$ lies in the normal subgroup, the right hand side is

$$
\rho_{1}(g) \rho\left(g^{-1} k g\right) \rho_{1}(g)=\rho_{1}(g) \rho_{1}\left(g^{-1} k g\right) \rho_{1}(g)=\rho_{1}(k)=\rho(k) .
$$

That is, the matrix $\rho_{1}(g) \rho_{2}\left(g^{-1}\right)$ centralises the irreducible representation $\rho$ so by Schur's Lemma (see Proposition 4 of [18] for example), this matrix must be central in $S L(2, \mathbf{C})$ and so lies in $\{ \pm I\}$.

This lemma demonstrates why passage to the $\operatorname{PSL}(2, \mathbf{C})$ character variety is required. As discussed in $\S 2$, the discrepency on lifting representations to $\mathrm{SL}(2, \mathbf{C})$ is a change of sign. Given this, a convenient way to use this lemma is the following, which has the same proof as 3.1:

Corollary 3.2. If two representations of $G$ into $\operatorname{PSL}(2, \mathbf{C})$ agree and are strongly irreducible on a subgroup of finite index, then they agree on $G$.

3.2. Before applying the results of $\S 3.1$ we introduce some notation. Suppose $p: M \rightarrow T$ is a covering of finite volume hyperbolic 3 -orbifolds. Then via restriction of representations, $p$ induces a map at the level of representation and character varieties, $p^{*}: Y_{0}(T) \rightarrow Y_{0}(M)$. Corollary 3.2 implies,

Theorem 3.3. Suppose that $p: M \rightarrow T$ is a covering of 1-cusped hyperbolic 3-orbifolds.

Then the induced map $p^{*}: Y_{0}(T) \rightarrow Y_{0}(M)$ is injective on characters of strongly irreducible representations. 
Proof. If the induced map were not injective then we could find a pair of distinct characters, and hence distinct strongly irreducible representations that agreed on a subgroup of finite index of $\pi_{1}^{\mathrm{orb}}(T)$. This contradicts Corollary 3.2.

If $T$ has a rigid cusp, so that by Theorem $2.1, Y_{0}(T)$ is a point, then Theorem 3.3 gives little information. However, in the presence of flexible cusps we have:

Theorem 3.4. Suppose that $p: M \rightarrow T$ is a covering of one cusped orbifolds and suppose that the cusp of $T$ is flexible.

Then the induced map $p^{*}: Y_{0}(T) \rightarrow Y_{0}(M)$ is a birational equivalence.

Proof. By Theorem 3.3, the map $p^{*}$ injects characters of strongly irreducible representations of $Y_{0}(T)$ into $Y_{0}(M)$. Now since hyperbolic Dehn surgeries on $T$ and $M$ give rise to a Zariski dense subset of characters of strongly irreducible representations in $Y_{0}(T)$ and $Y_{0}(M)$ respectively, to prove the theorem it suffices to show the map $p^{*}$ has nonzero degree at such points. To see this we proceed as follows.

Perform a genuine hyperbolic Dehn surgery on the cusp of $M$. This determines a character $\chi_{\rho} \in Y_{0}(M)$. We can extend the covering $p$ to a finite orbifold covering $M(\gamma) \rightarrow T(p(\gamma))$ so that in particular $T(p(\gamma))$ is a hyperbolic orbifold. Thus the pre-image of $\chi_{\rho}$ is non-empty.

We have therefore shown $p^{*}$ is a degree 1 map from $Y_{0}(T)$ to $Y_{0}(M)$ and therefore a birational equivalence.

We have thus proved Theorem 1.1 of the introduction:

Theorem 3.5. Suppose that $M_{1}$ and $M_{2}$ are 1-cusped hyperbolic 3-manifolds which cover a common orbifold with a flexible cusp.

Then $Y_{0}\left(M_{1}\right)$ is birational to $Y_{0}\left(M_{2}\right)$.

Restricting to the non-arithmetic case we can use 3.5 to make deductions about the entire commensurability class. The reason is that Margulis's characterization of arithmeticity implies that if $M_{i}(i=1,2)$ are non-arithmetic and commensurable, then there is a unique minimal element $T$ in the commensurability class of $M_{1}$ and $M_{2}$, see [11] and we therefore have finite sheeted coverings $M_{i} \rightarrow T$ for $i=1,2$. If we assume that $T$ does not have a rigid cusp we see that 3.5 implies Theorem 1.2 of $\S 1$ :

Theorem 3.6. Suppose that $M$ is a non-arithmetic 1-cusped hyperbolic 3-manifold for which the minimal element in the commensurability class has a flexible cusp.

Then $Y_{0}(M)$ is an invariant of the commensurability class of $M$.

The existence of a rigid cusp places strong restrictions on the cusps of manifolds in the commensurability class. For example, given an orbifold with a rigid cusp $C$, then any horospherical cross-section of a finite covering of $C$ has Euclidean modulus in $\mathbf{Q}(\sqrt{-1})$ or $\mathbf{Q}(\sqrt{-3})$ (see [13] or [16]). It is not hard to deduce from this (see [16]) that the invariant trace-field of such a manifold contains $\mathbf{Q}(\sqrt{-1})$ or $\mathbf{Q}(\sqrt{-3})$. With this we obtain the following. 
Theorem 3.7. Suppose that $M_{1}$ and $M_{2}$ are commensurable, non-arithmetic, 1-cusped hyperbolic 3-manifolds whose invariant trace fields do not contain either $\mathbf{Q}(\sqrt{-1})$ or $\mathbf{Q}(\sqrt{-3})$.

Then $Y_{0}\left(M_{1}\right)$ is birational to $Y_{0}\left(M_{2}\right)$.

Proof. The discussion above and the hypothesis on the invariant trace-field, shows that the minimal element in the commesurability class $T$ cannot have a rigid cusp. Theorem 3.4 implies the result.

Corollary 3.8. Suppose that $M$ is a non-arithmetic, 1-cusped hyperbolic 3manifold whose invariant trace field does not contain either $\mathbf{Q}(\sqrt{-1})$ or $\mathbf{Q}(\sqrt{-3})$. Then $Y_{0}(M)$ is an invariant of the commensurability class of $M$.

\section{Remarks:}

1. For knots in $S^{3}$ the figure-eight knot is the only arithmetic knot [16], and the existence of a rigid cusp quotient of non-arithmetic knot complements is related to the question of whether the minimal element in the commensurability class of a non-arithmetic hyperbolic knot complement is "smaller" than the orbifold obtained as the quotient of the knot complement by the group of orientation-preserving isometries (see [16] and [13]). In particular, apart from the figure-eight knot, the only knot complements that are known to cover an orbifold with a rigid cusp are the two dodecahedral knots of Aitchison and Rubinstein [1].

2. Theorem 3.4 and Corollary 3.5 have multi-cusp analogs. In this case the manifolds and quotient orbifold should have the same number of cusps, and in the latter case these should be flexible.

3.3. Some of the information contained in the invariant $Y_{0}$ can also be expressed in terms of detected slopes. Recall the following from [6].

Let $M$ be a 1-cusped hyperbolic 3-manifold of finite volume and let $\Gamma=\pi_{1}(M)$. For $\gamma \in \Gamma$ define the (regular) function $I_{\gamma}: X(M) \rightarrow \mathbf{C}$ by $I_{\gamma}(\chi)=\chi(\gamma)$. Let $\alpha$ be a boundary slope and $\beta$ a slope with $\{\alpha, \beta\}$ a generating set for the fundamental group of the peripheral torus. We say $\alpha$ is detected by a component $X_{1} \subset X(M)$ if there is a sequence of characters $\left\{\chi_{j}\right\} \in X_{1}$ of irreducible representations with $I_{\alpha}\left(\chi_{j}\right)$ remaining bounded, but $\left|I_{\beta}\left(\chi_{j}\right)\right| \rightarrow \infty$.

If there is a sequence of characters in a component $X_{1}$ which is bounded on all peripheral elements but still blows up on some element $\gamma$, we detect a closed embedded incompressible surface. In this case we say $X_{1}$ detects a closed incompressible surface.

As discussed in $\S 2$ the passage to $\operatorname{PSL}(2, \mathbf{C})$-charactetrs is only a matter of sign, and so the property of "blowing-up" will be preserved on passage to sequences of $\operatorname{PSL}(2, \mathbf{C})$-characters. Thus, we may use the above notation on the variety $Y(M)$. In this language our results show:

Corollary 3.9. Let $M_{1}$ and $M_{2}$ be as in Corollary 3.5. Then

(i) The number of boundary slopes detected by $Y_{0}\left(M_{1}\right)$ is the same as the number detected by $Y_{0}\left(M_{2}\right)$. 
(ii) If $Y_{0}\left(M_{1}\right)$ detects a closed incompressible surface so does $Y_{0}\left(M_{2}\right)$.

Proof. Let $T$ denote the common orbifold quotient, with $p_{i}: M_{i} \rightarrow T$ the quotient maps $(i=1,2)$. Now the peripheral subgroups of $M_{1}$ and $M_{2}$ have finite index in the peripheral subgroup of $T$. The main observation to make is that the property of a slope $\gamma_{i}$ blowing up or remaining bounded on $Y_{0}\left(M_{i}\right)$ implies the same for $p_{i}\left(\gamma_{i}\right)$ on $Y_{0}(T)(i=1,2)$. The fact that such a sequence of characters lifts to $Y_{0}(T)$ is again an application of Theorem 3.5.

Thus, if $\beta$ is a boundary slope detected on $Y_{0}\left(M_{1}\right)$ for $M_{1}$, then we can, by the above process associate a detected slope $\beta^{*}$ for $M_{2}$. This process defines a 1-1 map between the sets of detected slopes on $Y_{0}\left(M_{1}\right)$ and $Y_{0}\left(M_{2}\right)$. Furthermore, reversing this argument implies that from a slope detected on $Y_{0}\left(M_{2}\right)$, we get a slope detected on $Y_{0}\left(M_{1}\right)$. Hence we get a bijective correspondence between the set of detected boundary slopes on $Y_{0}\left(M_{1}\right)$ and $Y_{0}\left(M_{2}\right)$.

Case (ii) follows using a similar argument from the remarks above.

These results also allow us to deduce the existence of additional components in $Y(M)$; various statements are possible, the simplest being:

Corollary 3.10. Suppose $M_{1}$ and $M_{2}$ are commensurable 1-cusped hyperbolic 3-manifolds without closed incompressible surfaces. Suppose that $M_{1}$ has more detected slopes than $M_{2}$.

Then at least one of the $Y\left(M_{1}\right)$ or $Y\left(M_{2}\right)$ has more components that $Y(T)$.

Proof. By Lemma 3.1, each component of $Y(T)$ injects into some component of $Y\left(M_{1}\right)$ and since each component of the latter variety is a curve by the hypothesis on closed incompressible surfaces, this maps components of $Y(T)$ birationally into components of $Y\left(M_{1}\right)$. A similar statement holds for $M_{2}$.

Any component of $Y(T)$ produces the same number of boundary slopes in $M_{1}$ and $M_{2}$. Therefore the hypothesis shows that at least one (and possibly both) of $Y\left(M_{1}\right)$ or $Y\left(M_{2}\right)$ must have a component which does not appear in $Y(T)$.

\section{Examples}

In what follows we made considerable use of SnapPea [20] and Mathematica $^{T M}$. We assume familiarity with both. In particular the nomenclature mabc refers to that used in SnapPea for manifolds in the 5-tetrahedra census. Fundamental groups and peripheral data used are those given by SnapPea - for knots in $S^{3}$, this is usually not the usual meridian/longitude framing. We make no effort to detail the mathematica calculations. The main computations are straightforward applications of resultants in elimination theory. All examples considered had 2-generator fundamental groups. Letting $a$ and $b$ be generators, $X(M)$ (and $Y(M))$ are determined by three traces:

$$
P=\operatorname{tr}(a), \quad Q=\operatorname{tr}(b), \quad \text { and } \quad R=\operatorname{tr}(a b) .
$$

In the cases at hand, the calculations were simplified as $X_{0}(M)$ was given as a plane curve in terms of $P$ and $Q$. 


\section{Example: The complement of the knot $5_{2}(m 015)$ and $m 017$.}

The components $X_{0}$ and $Y_{0}$ for $m 015$ were computed in $\S 2.1$ - from there we see that $\widehat{X_{0}}(m 015)$ has genus 2 . The manifolds $m 015$ and $m 017$ have the same volume and invariant trace field being the cubic with one complex place and discriminant -23 . The two manifolds can be shown to be commensurable - for instance SnapPea shows they have a common 2 -fold cyclic cover. The variety $X_{0}(m 017)$ is defined by the plane curve $F(P, Q)=0$ where:

$$
F(P, Q)=P^{2} Q-Q+1 \text {. }
$$

In this example $R=\frac{1-3 P^{2}+P^{4}}{P^{3}-P}$. Note that multiplying the equation $P^{2} Q-Q+$ $1=0$ by $Q$, and setting $x=P Q, y=Q$, the curve is birational to $x^{2}=y^{2}-y$. This is a punctured sphere. Thus the $\operatorname{SL}(2, \mathbf{C})$-character varieties of these two manifolds are not birational. As computed in $\S 2.1$, the $\operatorname{PSL}(2, \mathbf{C})$-character variety is a punctured sphere.

Example: The manifolds $m 222$ and $m 224$.

These manifolds are knots in $S^{3}$, see [3]. The manifold $m 222$ being the complement of the knot $8_{20}$, and $m 224$ being the complement of the 11 crossing knot $11_{405}$, see [3]. They share the same volume, and have the same degree 5 invariant trace-field with two complex places and discriminant 5864. However these examples can be shown to be incommensurable using Theorem 1.1. To apply Theorem 1.1 first note that since the invariant trace-field has degree 5, the manifolds are non-arithmetic, and they cannot cover an orbifold with a rigid cusp. Hence to prove incommensurable, it suffices to check the (smooth models of the) curves $Y_{0}$ have different genus.

The components $X_{0}$ and $Y_{0}$ for these manifolds are:

$$
\begin{aligned}
X_{0}(m 222) & :=-1+4 P^{2}+3 P^{3}-4 P^{4}-P^{5}+P^{6}+P\left(-2+2 Q^{2}-Q^{4}\right)=0 \\
Y_{0}(m 222) & :=-1+4 P^{2}+3 P^{3}-4 P^{4}-P^{5}+P^{6}+P\left(-2+2 Z-Z^{2}\right)=0 \\
X_{0}(m 224) & :=-4 P+10 P^{3}-6 P^{5}+P^{7}+\left(-2+2 P^{2}\right) Q+\left(1-P^{2}\right) Q^{3}=0 \\
Y_{0}(m 224) & :=-4+10 Z-6 Z^{2}+Z^{3}+V(-2+2 Z)+V^{3}\left(Z-Z^{2}\right)=0
\end{aligned}
$$

First consider the curve for $Y_{0}(m 222)$ above. After a birational equivalence, this can be rewritten as:

$$
W^{2}=-P-P^{2}+4 P^{3}+3 P^{4}-4 P^{5}-P^{6}+P^{7},
$$

where $W=(P Z-P)$. Now the right hand side factors as

$$
P\left(-1-P+P^{2}\right)^{2}\left(-1+P+P^{2}\right),
$$

and so, as is easy to see, a further birational equivalence yields the plane cubic curve given by $y^{2}=x\left(x^{2}+x-1\right)$. This is an elliptic curve since the right hand side has distinct roots. Thus the genus of $\widehat{Y_{0}}(\mathrm{~m} 222)$ is 1 .

We now claim that the genus of $\widehat{Y_{0}}(m 224)$ is 4 so that the manifolds cannot be commensurable. The usual way to compute the genus is to take the plane 
curve and compute its genus as if it were smooth, and then subtract contributions from singular points. The following remarkable theorem (unpublished) of F. Rodriguez-Villegas gives a very simple way to compute the genus in many cases.

Theorem 4.1. Let $C$ be an irreducible plane curve which is smooth except perhaps at infinity. Assume $C$ is defined by the equation $F(x, y)=0$. Let $N$ denote the Newton polygon of $F$. Assume the edge polynomials of $N$ have no multiple roots. Then the genus of the smooth projective model of $C$ is the number of lattice points in the interior of $N$.

It is easily seen from above that the plane curve $Y_{0}(m 224)$ is smooth except at infinity and satisfies the edge conditions in Theorem 4.1. A count of interior lattice points gives 4 as required. We remark that using SnapPea these manifolds can also be shown to be incommensurable using their cusp volume.

Example: The figure-eight knot complement ( $m 004)$ and $m 022$.

Like the invariant-trace field, the curve $Y_{0}$ is not a complete invariant of commensurability. For example, the two manifolds in question are not commensurable, the invariant trace-fields being $\mathbf{Q}(\sqrt{-3})$ and a totally imaginary quartic field respectively. However, the varieties $X_{0}(m 004)$ and $X_{0}(m 022)$ are birational, as are the varieties $Y_{0}(m 004)$ and $Y_{0}(m 022)$. The curve $X_{0}(m 004)$ is an elliptic curve of conductor 40 (see [10]) and the latter is a sphere.

Example: The complement of the knot $7_{4}, m 006$ and $m 007$.

Using SnapPea, it can be checked these three manifolds are commensurable. The volumes of $m 006$ and $m 007$ are the same, approximately $2.5689706009 \ldots$ and the volume of $S^{3} \backslash 7_{4}$ is twice this. The invariant trace-field is cubic of discriminant -59 . Thus any orbifold quotient of these manifolds has a flexible cusp. The $\operatorname{PSL}(2, \mathbf{C})$ curve which is an invariant of the commensurability class has genus 0 . The main interest in these examples is that the character variety $Y\left(S^{3} \backslash 7_{4}\right)$ has an additional component containing an irreducible representation. The character varieties for $m 006$ and $m 007$ do not. This is reflected in the fact that $S^{3} \backslash 7_{4}$ has three detected boundary slopes (cf., Corollary 3.10).

Another interesting feature about the manifolds $m 006$ and $m 007$ is that it appears that the number of boundary slopes is exactly 2 - not just strict boundary slopes. If this is not the case then there would be an undetected strict boundary slope, which seems unlikely. The fact that at least 2 slopes are detected on $X(M)$ (or $Y(M))$ is proved in [7]. The A-polynomials for these two manifolds are given below:

$$
\begin{aligned}
& A_{m 006}(\mu, \lambda)=\lambda^{5} \mu^{2}+\mu^{3}+\lambda\left(-1+2 \mu^{2}\right)+\lambda^{2}\left(-\mu-\mu^{3}\right)+\lambda^{3}\left(-\mu^{2}-\mu^{4}\right) \\
& +\lambda^{4}\left(2 \mu^{3}-\mu^{5}\right) \\
& A_{m 007}(\mu, \lambda)=-\lambda+2 \lambda \mu^{2}+\mu^{3}-\lambda^{2} \mu^{3}+\lambda \mu^{4}-\lambda^{3} \mu^{4}+\lambda^{2} \mu^{5}-\lambda^{4} \mu^{5} \\
& -2 \lambda^{3} \mu^{6}+\lambda^{3} \mu^{8} \text {. }
\end{aligned}
$$

From [4] the slopes of the edges of the Newton polgon are boundary slopes for the manifold. These account for all detected slopes. The boundary slopes for 
$m 006$ are $\{-3,1 / 2\}$ and for $m 007$ are $\{-3,5 / 3\}$. In both these cases, -3 -Dehn surgery gives a manifold with positive first betti number. All other slopes are rational homology spheres.

\section{References}

[1] I.R. Aitchison and J.H. Rubinstein, Combinatorial cubings, cusps and the dodecahedral knots, Topology '90 (Columbus, OH, 1990), 17-26, Ohio State Univ. Math. Res. Inst. Publ., 1, De Gruyter, Berlin, 1991.

[2] S. Boyer and Z. Zhang, On Culler-Shalen seminorms and Dehn filling, Ann. of Math. (2) 148 (1998), 737-780.

[3] P.J. Callahan, J.D. Dean, and J.R. Weeks, The simplest hyperbolic knots, preprint.

[4] D. Cooper, M. Culler, H. Gillet, D.D. Long, and P.B. Shalen, Plane curves associated to character varieties of 3-manifolds, Invent. Math. 118 (1994), 47-84.

[5] M. Culler, C. Gordon, J. Luecke, and P.B. Shalen, Dehn surgery on knots., Ann. of Math. (2) 125 (1987), 237-300.

[6] M. Culler and P.B. Shalen, Varieties of group representations and splittings of 3manifolds, Ann. of Math. (2) 117 (1983), 109-146.

[7] M. Culler and P.B. Shalen, Bounded separating incompressible surfaces in knot manifolds, Invent. Math. 75 (1984), 537-545.

[8] W.D. Dunbar and G.R. Meyerhoff, Volumes of hyperbolic 3-orbifolds, Indiana Univ. Math. J. 43 (1994), 612-637.

[9] F. Gonzalez-Acuna and J.M. Montesinos, On the character variety of group representations in SL(2, C) and PSL(2, C), Math. Z. 214 (1993), 627-652.

[10] D.D. Long and A.W. Reid, Integral points on character varieties, preprint.

[11] G.A. Margulis, Discrete subgroups of semisimple lie groups, Ergebnisse der Mathematik und ihr Grenzgebeite (3), 17, Springer-Verlag, Berlin, 1991.

[12] D. Mumford, Algebraic geometry I: Complex projective varieties, Grundlehren der Mathematischen Wissenschaften, No. 221., Springer-Verlag, Berlin-New York, 1976.

[13] W.D. Neumann and A.W. Reid, The arithmetic of hyperbolic manifolds, Topology '90 (Columbus, OH, 1990), 273-310, Ohio State Univ. Math. Res. Inst. Publ., 1, De Gruyter, Berlin, 1991.

[14] W.D. Neumann and D. Zagier, Volumes of hyperbolic 3-manifolds, Topology 24 (1985), $307-332$.

[15] C. Petronio and J. Porti, Negatively oriented ideal triangulations and a proof of Thurston's hyperbolic Dehn filling theorem, preprint, math.GT/9901045.

[16] A.W. Reid, The arithmeticity of knot complements, J. London Math. Soc. (2) 43 (1991), 171-184.

[17] A.W. Reid, A note on trace-fields of Kleinian groups, Bull. London Math. Soc. 22 (1990), 349-352.

[18] J.P. Serre, Linear representations of finite groups, Graduate Texts in Mathematics, Vol. 42., Springer-Verlag, New York-Heidelberg, 1976.

[19] W.P. Thurston, The geometry and topology of 3-manifolds, Princeton University mimeographed notes (1979).

[20] J. Weeks, Snap Pea: A computer program for creating and studying hyperbolic 3manifolds, http://www.northnet.org/weeks/

Department of Mathematics, University of California at Santa Barbara, Santa BARBARA, CA 93106

E-mail address: long@math.ucsb.edu

Department of Mathematics, University of Texas at Austin, Austin, TX 78712

E-mail address: areid@math.utexas.edu 